\title{
Near-field scattering by dielectric spheroidal particles with sizes on the order of the illuminating wavelength
}

\author{
Manuel J. Mendes, * Ignacio Tobías, Antonio Martí, and Antonio Luque \\ Instituto de Energla Solar, Universidad Politécnica de Madrid, Avenida Complutense s/n 28040, Madrid, Spain \\ *Comesponding author: manuel mendesàies-def.upmes
}

\begin{abstract}
We present a theoretical study of electric field scattering by wavelength-sized spheroids. The incident, internal, and scattered fields are computed analytically by a spheroidal coordinate separation-of-variables solution, assuming axially incident monochromatic illumination. The main solrces of possible numerical errors are identified and an additional point-matching procedure is implemented to provide a built-in test of the validity of the results. Numerical results were obtained for prolate and oblate particles with particular aspect ratios and sizes, and a refractive index of 1.33 relative to the surrounding medinm. Special attention is paid to the characteristics of the near-field in close proximity to the spheroids. It is shown that particles with sizes close to the incident wavelength can produce high field enhancements whose spatial location and extension can be controlled by the particle geometry.
\end{abstract}

OCIS codes: $290.5850,260.2160,260.2110$.

\section{INTRODUCTION}

The analysis of electromagnetic (EM) wave scattering by spheroidal particles has been of increasing interest since the geometry of many objects of practical use can be approximated by a spheroidal shape, and the fields can be obtained analytically. The exact analytical solution of the Maxwell's equations for the plane-wave scattering by a homogeneous sphere of arbitrary size was obtained by Mie [1], using the method of separation of variables. This problem was first fully solved for the more general case of a homogeneous spheroid by Asano and Yamamoto [2], for any angle of incidence. Their approach consists of separating the vector wave equations in spheroidal coordinates and expanding them in terms of the spheroidal wave functions. The conditions of continuity of the tangential fields across the surface of the spheroid generate a set of simultaneous linear equations that can be solved for the set of unknown expansion coefficients. The coefficients are determined using the orthogonality integral approach, similar to the method used here [3].

Theoretically, the solution obtained in this way is exact. However, this does not happen in practice as it is not possible to include terms of infinite order in the series expansions and due to unavoidable computational inaccuracies. These issues become more significant when considering particles with sizes close or above the illuminating wavelength. In this paper the main sources of numerical error in the method are identified, and the best ways to reduce them are described based on the previous literature and our empirical tests. In addition, a simple built-in test procedure is described to check on the validity of the computed results. This makes use of a boundary condition (BC) point-matching (PM) strategy that can refine the initial solution.
Until the 1990 s most of the literature on analytical solutions of scattering by spheroidal particles was mainly concerned with the study of far-field quantities, such as electric field intensities at infinity and scattering cross sections [2-6]. In recent years there has been a growing shift of attention toward the near-field, located typically within a region close to the scatterer surface of about its size. This interest was sparked by technological advances in the area of nano-optics $[7,8]$ through novel techniques that can fabricate and probe (e.g., scanning near-field microscopy) sub-wavelength structures, and also due to the appearance of a new range of nanotechnological applications that can benefit from the enhanced local fields in the vicinity of scattering particles: high-resolution spectroscopy imaging (e.g, luminescence enhancement [9]), localized sensing methods (e.g., surface enhanced Raman spectroscopy [10]), and novel photovoltaic cell designs (e.g., intermediate-band cells [11]), among many others.

So far most of the analyses of EM scattering aimed at near-field applications consider objects that are either so small compared to the wavelength that the electrostatic (ES) (non-retarded) approximation becomes applicable, or particles with a spherical geometry that can be treated with Mie theory [8]. Studies that go beyond these limitations, dealing with non-spherical objects with sizes on the order of or bigger than the wavelength, usually employ numerical grid-based approaches such as the finite difference time-domain [12] and finite element [13] methods. With mesh approaches it is usually difficult to resolve high field gradients that occur at the surface of particles that scatter highly resonantly with the incoming light. Therefore, the design of each mesh must be well defined and refined for each particle structure. In particular the optical properties of particles with sizes close to the wave- 
length are very dependent on their geometry, material, and surrounding medium. Thus, to model the optical response of particles in the size ranges of interest in this work, it is beneficial to use analytical techniques since the fields can be calculated everywhere without the need to cope with finite mesh resolution errors. The main drawback of using analytical methods is that only "round shapes" can be considered [14], i.e., shapes without corners like ellipsoids, infinite cylinders, toroids, etc.

The solution of EM scattering by objects which are small compared to the wavelength becomes highly simplified, relative to the full arbitrary-size solution, since retardation effects can be neglected and only one scattering mode - the dipole mode-is excited. Therefore, scatterers in this regime produce very similar field patterns (dipolar-like), independently of their geometry [11,14]. When the particle size approaches the wavelength, higher order modes are excited producing additional features in the field pattern which are highly dependent on the scatterer geometry. In this paper we provide a numerical study of scattering by spheroidal particles with sizes comparable to the wavelength, which are seen to be able to produce scattered fields with magnitudes significantly higher than the incident field. Besides, unlike subwavelength particles in the dipolar regime, the position and extension of the field enhancement can be controlled according to the particle geometry.

Here we study how the electric field pattern is influenced by the scatterer size and shape for spheroids with a real index of refraction 1.33 times that of the medium in which they are embedded. This refractive index is chosen to allow a comparison between results obtained here and previous studies presenting scattering patterns of spheroids calculated by distinct methods $[2,3,12,15-17]$. Such a refractive index has been widely adopted since it corresponds to water in air, and therefore the computed results can be used for atmospheric simulation. Nevertheless, these results can also be useful for any of the previously referred applications by making an appropriate choice of the scatterer material. For instance, the relative index of germanium particles in a gallium arsenide medium is close to 1.3 in the solar infrared range, which can be of interest for the implementation in photovoltaic devices.

Furthermore, in this paper different size regimes are identified for the distinct types of near-field distributions produced. Particular attention is drawn to the polarization characteristics in each regime. It is important to distinguish between the polarization components, longitudinal (parallel to the wavevector $\mathbf{K}$ ) and transverse (orthogonal to $\mathbf{K}$ ), when considering the interaction between the scattered light and a material in the near-field of the scattering particle [18], as shall be described in more detail in Subsection 3.B.

\section{DESCRIPTION OF THE SEPARATION-OF- VARIABLES METHOD}

We consider the scattering of a plane, linearly polarized monochromatic wave by a spheroidal particle of arbitrary size whose axis of revolution is oriented along the incident wave direction. Both the particle material and the surrounding medium are homogeneous, isotropic, and non- magnetic. The exact solution is obtained by expanding the incident, scattered, and internal fields in terms of an infinite sum of spheroidal vector wave functions whose coefficients are determined from the $\mathrm{BCs}$ at the spheroidmedium interface.

Here we adopt a development [3,5] of the method of Asano and Yamamoto [2] that employs a formulation where the column matrix of the series coefficients of the scattered field is obtained from the matrix of the series coefficients of the incident field by means of a matrix transformation. The coordinate system used is either prolate or oblate spheroidal, depending on the scatterer geometry. The definition of the spheroidal coordinates $(\eta, \xi, \phi)$ is given in the Appendix. These are orthogonal curvilinear coordinates which allow the separation of variables to obtain the eigenfunctions of the scalar potential wave equation. In the prolate system these eigenfunctions are [19]

$$
\psi_{\varepsilon_{m}}^{(i)}(C ; \eta, \xi, \phi)=S_{m n}(C, \eta) R_{m m}^{(i)}(C, \xi) \times\left\{\begin{array}{c}
\cos (m \phi) \\
\sin (m \phi),
\end{array}\right.
$$

where $S_{m n}(C, \eta)$ is the spheroidal angular harmonic of the first kind, with order $m$ and degree $n$, and $R_{m n}^{(i)}(C, \xi)$ is the spheroidal radial harmonic of the $i$ th kind. The $\phi$ dependence is equal to the product of either a cosine (even) or a sine (odd), indexed by the subscripts $e$ and $o$, respectively. The spheroid size parameter $(C)$ is defined by

$$
C=\frac{K d}{2},
$$

where $K$ is the wavevector magnitude in the corresponding medium and $d$ is the particle interfocal distance. From the eigenfunctions $\psi_{e_{m n}}^{(i)}$ we can obtain $[3,19]$ the spheroidal vector wave functions $\mathbf{M}_{e_{m n}}^{q(i)}=\nabla \times\left[\psi_{e_{m n}}^{(i)} \hat{\mathbf{q}}\right]$ and $\mathbf{N}_{e_{m n}^{q(j)}}^{q(j)}=K^{-1} \nabla \times \mathbf{M}_{e_{m n}}^{q(i)}$, with $\hat{\mathbf{q}}=\hat{\mathbf{x}}, \hat{\mathbf{y}}, \hat{\mathbf{z}}$. When the incident light direction is collinear with the symmetry axis of the spheroid, the series coefficients vanish for $m \neq 0$ for the expansions of the polarized waves in terms of vector functions with $q=x, y$, whereas the coefficients of the expansions in $q=z$ functions are only nonzero for $m=1$ [19]. Thus, the summation over $m$ in the fields' expansions reduces to a single $m$ term. The expansions are therefore represented by a single sum over the degree $n$ [6]. The expressions of the vector wave functions used for axial incidence are given in the Appendix.

Figure 1 shows a schematic of the coordinate system used here. At the axial incidence the exciting plane-wave can be resolved by a single polarization component, by virtue of symmetry. This wave is then assumed to propagate along the negative $z$ direction with the electric field $\mathbf{E}_{0}$ parallel to $y$, and magnetic field $\mathbf{H}_{0}$ along the positive $x$ axis. The electric field unit considered in this work is $E_{0}$. As such, the illuminating wave has unitary electric field amplitude and can be described by [19]

$$
\begin{aligned}
\mathbf{E}_{0}= & \frac{1}{K_{0}} \sum_{n=0}^{\infty} A_{n} \mathbf{M}_{e(n)}^{x i 1 j}\left(C_{0} ; \eta, \xi, \phi\right), \quad \mathbf{H}_{0}=\frac{i}{K_{(} Z_{(n)}} \sum_{n=0}^{\infty} A_{n} \mathbf{N}_{e(n)}^{x i 1)} \\
& \times\left(C_{0} ; \eta, \xi, \phi\right)
\end{aligned}
$$

where $K_{0}=2 \pi / \lambda$ (with $\lambda$ being the wavelength in the me- 


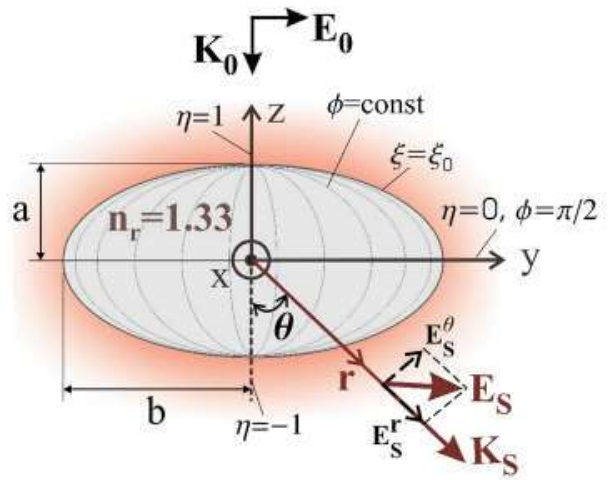

Fig. 1. (Color online) Coordinate system for scattering by a spheroid. The particles considered in this work are dielectric with an index of refraction of $n_{r}=1.33$ relative to the surrounding medium. The surfaces $\xi=$ const are spheroids confocal with the particle, and $\xi_{0}$ coincides with the particle surface. The direction of illumination $\left(\mathbf{K}_{0}\right)$ is collinear with the spheroid axis of symmetry $(z)$. A scattered wave in the $z y$ plane is shown, making an angle $\theta$ with the negative $z$ axis. It propagates along $\mathbf{K}_{\mathbf{S}}$ and its electric field $\left(\mathbf{E}_{\mathrm{S}}\right)$ can have a component longitudinal $\left(\mathbf{E}_{\mathrm{S}}^{\mathbf{r}}\right)$ and transverse $\left(\mathbf{E}_{\mathrm{S}}^{\theta}\right)$ relative to the propagation direction.

dium outside the particle), $C_{0}=K_{0} d / 2$, and $Z_{0}=\sqrt{\mu_{0} / \varepsilon_{0}}$ is the characteristic impedance of free space. The coefficients $A_{n}$ of the incident wave expansion are $A_{n}$ $=2 i^{n-1} S_{0 n}\left(C_{0}, 1\right) / \int_{-1}^{1} S_{0 n}\left(C_{0}, \eta\right)^{2} \mathrm{~d} \eta$.

The scattered field is described by vector wave functions of the fourth kind to satisfy the radiation condition at infinity [6],

$$
\begin{aligned}
\mathbf{E}_{\mathbf{S}} & =\sum_{n=0}^{\infty}\left[\alpha_{n} \mathbf{M}_{e 0 n}^{+(4)}\left(C_{0} ; \eta, \xi, \phi\right)+\beta_{n+1} \mathbf{M}_{e 1, n+1}^{z(4)}\left(C_{0} ; \eta, \xi, \phi\right)\right], \\
\mathbf{H}_{\mathbf{S}} & =\frac{i}{Z_{0}} \sum_{n=0}^{\infty}\left[\alpha_{n} \mathbf{N}_{e 0 n}^{+(4)}\left(C_{0} ; \eta, \xi, \phi\right)+\beta_{n+1} \mathbf{N}_{e 1, n+1}^{z(4)}\left(C_{0} ; \eta, \xi, \phi\right)\right],
\end{aligned}
$$

where

$$
\mathbf{M}_{e 0 n}^{+(i)}(C ; \eta, \xi, \phi)=\frac{1}{2} \mathbf{M}_{e 0 n}^{x(i)}(C ; \eta, \xi, \phi)
$$

and

$$
\mathbf{N}_{e 0 n}^{+(i)}(C ; \eta, \xi, \phi)=\frac{1}{2} \mathbf{N}_{e 0 n}^{x(i)}(C ; \eta, \xi, \phi) .
$$

Finally, the transmitted field inside the spheroid $\left(\xi<\xi_{0}\right)$ can be expressed as [3]

$$
\begin{aligned}
\mathbf{E}_{\mathrm{I}} & =\sum_{n=0}^{\infty}\left[\gamma_{n} \mathbf{M}_{e 0 n}^{+(1)}\left(C_{I} ; \eta, \xi, \phi\right)+\delta_{n+1} \mathbf{M}_{e 1, n+1}^{z(1)}\left(C_{I} ; \eta, \xi, \phi\right)\right], \\
\mathbf{H}_{\mathrm{I}} & =\frac{i}{Z} \sum_{I n=0}^{\infty}\left[\gamma_{n} \mathbf{N}_{e 0 n}^{+(1)}\left(C_{I} ; \eta, \xi, \phi\right)+\delta_{n+1} \mathbf{N}_{e 1, n+1}^{z(1)}\left(C_{I} ; \eta, \xi, \phi\right)\right],
\end{aligned}
$$

where $C_{I}=n_{r} C_{0}$, with $n_{r}$ being the ratio of the refractive indices of the particle and the medium. The expressions given in this section are written with respect to the prolate coordinate system, but they are identical for the oblate system with the interchanges $c \rightarrow-i c$ and $\xi \rightarrow i \xi$.
The sets of unknown expansion coefficients $\alpha_{n}, \beta_{n+1}, \gamma_{n}$, and $\delta_{n+1}$, in expressions (3) and (4) for the scattered and internal fields, can be determined by applying the $\mathrm{BCs}$ at the surface $\left(\xi=\xi_{0}\right)$. These require the continuity of the tangential electric and magnetic field components,

$\xi=\xi_{0}: \quad\left\{\begin{array}{r}E_{0}^{\eta}=E_{I}^{\eta}-E_{S}^{\eta}, \\ E_{0}^{\phi}=E_{I}^{\phi}-E_{S}^{\phi}, \\ H_{0}^{\eta}=H_{I}^{\eta}-H_{S}^{\eta}, \\ H_{0}^{\phi}=H_{I}^{\phi}-H_{S}^{\phi} .\end{array}\right.$

The four sets of unknowns are of infinite number and coupled to each other; therefore in practice it is impossible to obtain the exact solution. However, a sufficient computational accuracy can be achieved by properly choosing the truncation number $(N)$ in the fields' expansions (2)-(4). In this work we take

$$
N=\text { integer }\left(K_{0} \alpha+4\right) \text {, }
$$

with $\alpha$ being the longest semi-axis of the spheroidal particle. This was seen to be the most reasonable choice based on the literature $[3,5,6]$ and empirical tests performed by the authors.

\section{A. Determination of the Expansion Coefficients by the Orthogonality Integral Approach}

The following methodology $[3,5,6]$ is used to find the unknown coefficients $\alpha_{n}, \beta_{n+1}, \gamma_{n}$, and $\delta_{n+1}$ :

(1) The $\phi$ dependence of the BCs (5) is removed by applying the orthogonality properties of the trigonometric functions. However, in the particular case of axial incidence this is not necessary since all vector wave functions in Eqs. (5a) and (5d) have a $\sin (\phi)$ factor, and those in Eqs. (5b) and (5c) have a $\cos (\phi)$ factor, which can therefore be readily eliminated.

(2) The $\eta$ dependence from the BCs is removed by making use of the angular function $\left(S_{m n}\right)$ orthogonality properties. $S_{m n}(C, \eta)$ are expansions of Legendre functions, so their orthogonality comes from the Legendre polynomial orthogonality relations. The approach consists of writing $N+1$ equations for each BC in Eqs. (5), with each equation being multiplied by a distinct $S_{0, n}$ term (where $n$ $=0,1,2, \ldots, N)$. The $4(N+1)$ equations constructed in this way are then integrated in $\eta$ over its domain (from -1 to 1); the orthogonality properties are applied to compute the integrals.

(3) The system of $4(N+1)$ equations, now independent of $\phi$ and $\eta$, is solved for the $4(N+1)$ unknowns (the expansion coefficients). This system can be written in a matrix form,

$$
\mathrm{QA}=\mathrm{R} \mathbf{X},
$$

where $Q$ is a $4(N+1) \times(N+1)$ matrix with the values of Eq. (2) that multiply the incident field coefficients $A_{n}$ and the calculated integrals in $\eta$ from the previous step. The $A_{n}$ coefficients are included in the $(N+1)$ column vector $\mathbf{A}$. The matrix $\mathrm{R}$ has a dimension of $4(N+1) \times 4(N+1)$ and 
contains the values in Eqs. (3) and (4) that multiply the unknown coefficients with the integrals in $\eta$ evaluated at $\xi=\xi_{0}$. The unknowns $\alpha_{n}, \beta_{n+1}, \gamma_{n}$, and $\delta_{n+1}$ are included in $\mathbf{X}$, arranged in a column vector of dimension $4(N+1)$. The vector $\mathbf{X}$ is determined by inverting the $R$ matrix,

$$
\mathbf{X}=\mathrm{R}^{-1} \mathrm{QA} \text {. }
$$

The computational environment used to perform the calculations is Mathematica 7.0, suitable due to its symbolic and high-precision numerical capabilities, as well as its large library of built-in routines that include packages for the computation of spheroidal harmonics $[3,20,21]$.

The results presented in this work were generated in an Intel Duo Core central processing unit machine with $2.33 \mathrm{GHz}$ and 2 Gbytes of random access memory. The total time needed to calculate the expansion coefficients with the method described is mainly dependent on the number of orders $(N)$ used. For a low truncation number $(N \leq 7)$ the total time, in minutes, is roughly equal to $N / 2$. For higher $N$ it scales approximately with $N^{2}$.6 for prolates and $N^{3}$ for oblates. Most of this time is spent in the computation of the overlap integrals in step (2).

\section{B. Identification of Possible Numerical Error Sources} Once the coefficient vector $\mathbf{X}$ is obtained the problem is solved, since the $\mathbf{E}$ and $\mathbf{H}$ fields are known everywhere [Eqs. (2)-(4)]. This result should give an accurate solution for the fields as long as the number of orders $N$ is sufficiently high. However in practice this may not happen, no matter how big $N$ is, due to computational inaccuracies that can occur at every step of the program. These errors tend to become more significant for higher orders, which may cause severe problems when attempting to consider particle sizes close or above the illuminating wavelength. In order to prevent such issues, particular attention should be paid to the following important points in the method:

(1) Determination of the eigenvalues $\lambda_{m n}(C)$ of the spheroidal angular and radial harmonics-There are several methods that can be used to evaluate the eigenvalues [3]; of these the one found to be more precise, for any size parameter $C$, and consistent with tabulated published results $[19,20,22]$ is the tridiagonal matrix method $[21,23]$. This is the method employed by the Mathematica 7.0 routine SpheroidalEigenvalue.

(2) Calculation of the spheroidal harmonics $S_{m n}$ and $R_{m y}$-According to our numerical tests and comparison with the literature results, both of these functions can be accurately computed with the Mathematica 7.0 built-in routines based on the code of Falloon et al. [20]. However, the calculation of the $S_{m m}$ functions becomes relatively slow for large and complex size parameters $(C)$. The routine used in this work, based on the code developed by $\mathrm{Li}$ et al. [3,21], calculates the angular functions considerably faster, maintaining a high numerical precision as shown in Fig. 2.

(3) Inversion of the matrix $\mathrm{R}$ in $\mathrm{Eq}$. (8)-For problems involving large particles that hence require many $N$ orders [according to Eq. (6)], the matrix $R$ can become very sparse with values spanning several orders of magnitude. In such cases a simple inversion routine performed by a standard algorithm (such as Gaussian elimination or oth-

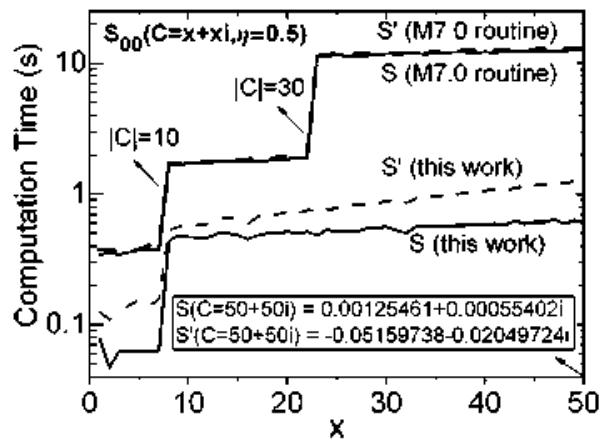

Fig. 2. (Color online) Computational time to calculate the angular harmonics $S_{m n}(C, \eta)$ and $S^{\prime}=\mathrm{d} S / \mathrm{d} \eta$, with $m=n=0, \eta=0.5$, and $C=x+x i$, as a function of $x$. The calculations were performed using the Mathematica 7.0 built-in routine and the routine used in this work. For all $x$ values both oodes give the same results for $S$ and $S^{\prime}$ up to the 14th decimal place. The inset shows the results for $x=50$, which match those presented in [3].

ers) may not provide an accurate enough result. Therefore an iterative correction algorithm that refines the output of the matrix inversion should be used. The algorithm given in Section 2.5 of [24] was implemented here. This routine iteratively reduces the value of the inversion error defined as $\varepsilon=\left\|R R^{-1}-I\right\|$, with I being the identity matrix. Typically $\varepsilon$ can be reduced by 1 or 2 orders of magnitude with as few as five iterations, with the total computational time being practically negligible (on the order of $0.1 \mathrm{~s}$ ).

\section{Boundary Point-Matching Test}

Further error can be induced by the limited precision of any computer calculation. Therefore, it is advisable to implement an additional routine able to check the correctness of the solution of $\mathrm{Eq}$. (8) and, if possible, to refine it. As such, we included in the code a simple iterative refinement cycle based on the $\mathrm{BC}$ point matching (PM) $[4,25,26]$.

An alternative way of determining the $4(N+1)$ expansion coefficients $\alpha_{n}, \beta_{n+1}, \gamma_{n}$, and $\delta_{n+1}$ to that described in Subsection 2.A would be to directly solve the BCs (5) for the tangential components of the fields at a set of $M$ points picked along the spheroid surface. The number of points chosen has to be at least $M_{\min }=2(N+1)$ to ensure that the number of obtained equations is equal to the number of unknown coefficients in the expansions.

To avoid ill-conditioned $\mathrm{BC}$ matrices with the PM, it is important that the $M$ points are well distributed along the surface. The authors in [27] found it advantageous to place more points near the spheroid axis $(\eta= \pm 1)$ and fewer at $\eta \approx 0$, while others [28] implemented an algorithm to determine the optimal point positions. The most favorable location of the points depends on the spheroid geometry; therefore it is advantageous to use more than the minimum number of points $\left(M>M_{\min }\right.$ ) and solve for the coefficients providing the minimum mean square error over the point set-the least-squares solution $[4,29]$. The more points are used the more the error is reduced, and the less sensitive the solution becomes to the chosen positions of the points. In our code the points are positioned at equally spaced intervals from $\eta=-1$ to $\eta=1$, as in [17]: half of them at $\phi=0$ and the other half at $\phi$ 
$=\pi / 2$. The total number of fitting points $M$ was taken to be about twice the number of minimum points $M=4(N$ +1 ), as suggested in previous applications of the method [4]. For each point the tangential internal and scattered vector wave functions in Eqs. (3) and (4) are calculated, and the values are inserted in a matrix $A$ with a dimension of $2 M \times 4(N+1)$. According to the $\mathrm{BCs}(5)$, this matrix multiplied by the solution of Eq. (8) should satisfy

$$
\mathrm{AX}=\mathbf{B} \text {, }
$$

where $\mathbf{B}$ is a vector of dimension $2 M$ determined by the two tangential components of the incident field at the picked surface points. Equation (9) is, in general, not exactly fulfilled due to possible errors in the computation of $\mathbf{X}$. The correct equation is therefore:

$$
\mathbf{A}\left(\mathbf{X}_{\text {correct }}+\mathbf{X}_{\text {error }}\right)=\mathbf{B}+\mathbf{d B} \text {, }
$$

where $\mathbf{X}_{\text {error }}$ is the error in $\mathbf{X}$ which we want to find. The vector $\mathbf{d B}$ is the error on the right-hand side of Eq. (9) and can be calculated as $\mathbf{d B}=\mathbf{A X}-\mathbf{B}$. From Eqs. (9) and (10) we get

$$
\mathrm{AX}_{\mathrm{error}}=\mathbf{d B}
$$

Since $\mathbf{A}$ and $\mathbf{d B}$ are known, $\mathbf{X}_{\text {error }}$ can be determined from the least-squares solution of Eq. (11). Then finally a more correct solution $\mathbf{X}_{\text {correct }}$ is obtained by

$$
\mathbf{X}_{\text {correct }}=\mathbf{X}-\mathbf{X}_{\text {error }} \text {. }
$$

This solution is further refined in an iteration cycle which improves the least-squares operation. At each iteration the result of Eq. (12) is taken as the new initial X solution; it is inserted back into Eq. (9) and the previous calculations are repeated. The iterations then proceed until convergence is achieved.

From the tests performed, the authors have concluded that the final $\mathbf{X}_{\text {correct }}$ solution does not bring a considerable improvement to the initial $\mathbf{X}$ solution from Eq. (8) if $\mathbf{X}$ is determined with the advisable amount of $N$ (or more) orders given in Eq. (6). If fewer orders are used, this additional PM refinement can improve the result. However, for particle sizes on the order of or bigger than the wavelength, the improvement is never as good as that which would be obtained with the correct number of $N$ orders in Eq. (6), no matter how many $M$ points are used. Therefore, the addition of this PM algorithm should not be used to compensate for using a lower number of $N$ orders than Eq. (6) in the method of Subsection 2.A. The main reason why it was implemented in the program is because it provides an automatic check on the correctness of the expansion coefficients result. The computational time required for this PM test is on the same order of the time spent in calculating the expansion coefficients with the orthogonality integral approach described in Subsection 2.A.

\section{PRESENTATION OF RESULTS}

The way in which the scatterer geometry influences the field around it changes considerably according to the size of the particle relative to the illuminating wavelength $(\lambda)$. Three main scattering regimes can be distinguished: the "small particle" or electrostatic (ES) regime, the "big par-

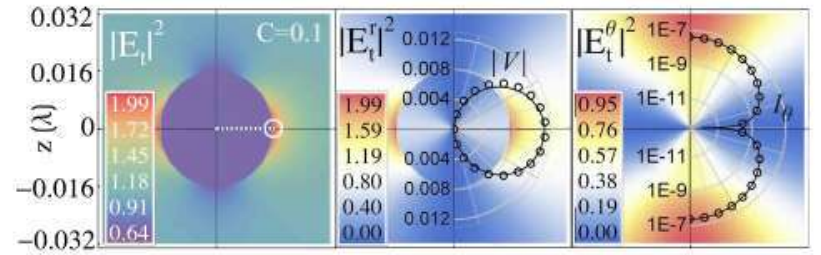

Fig. 3. (Color online) Electric field distribution in the $y z$ plane of a sphere with $n_{r}=1.33$. Left-Total field $\left(\mathbf{E}_{\mathrm{t}}\right)$ magnitude squared. For spheres the size parameter is $C=2 \pi R / \lambda$, where $R$ is the radius. The white circle is at the spot of maximum intensity. Center-Squared magnitude of the longitudinal component $\left(\mathbf{E}_{t}^{\mathbf{r}}\right)$. The polar plot depicts the ES potential magnitude $(|V|)$ at the particle surface $(r=R)$. Right_Squared magnitude of the transverse component $\left(\mathbf{E}_{t}^{\theta}\right)$. The polar plot shows the far-field intensity function $I_{\theta}\left[\mathrm{Eq}\right.$. (14)]. In the plots of $|V|$ and $I_{\theta}$ the circular dots correspond to the values obtained with the ES (or Rayleigh) approximation $[8,14]$.

ticle" regime described by macroscopic geometrical optics, and in between an intermediate regime for particle sizes on the order of $\lambda$. This intermediate regime, defined by some authors as the domain of physical optics [30], will be the focus of this paper using the previously described numerical code.

Most of the literature on analytical solutions of EM scattering by spheroidal particles, with sizes comparable to $\lambda$, mainly analyzes far-field quantities. However, the internal field and near-field around such particles contain additional physically rich features worth analyzing in more detail $[12,16]$. The length unit used is $\lambda$, making the results given here dimensionless and therefore independent of the particular wavelength of illumination.

\section{A. Numerical Verification}

The EM code used in this work was extensively checked with known analytical and numerical solutions available in the literature. Some examples of such comparisons are given along the presentation of the results. In the next subsection we start by looking at the electric field distribution around a small sphere in the ES regime, which is compared in Fig. 3 with the analytical ES approximation [14]. In the following subsections results are shown for bigger spheroids with certain shapes selected to illustrate the main characteristics of the intermediate regime. These are compared with curves obtained numerically by Asano and Yamamoto [2] in Figs. 4-7. Finally, the inset in Fig. 10 shows a comparison with Mie theory [1] considering a prolate with $a \approx b=\lambda$. In all cases the numerical results obtained by us match the known solutions.

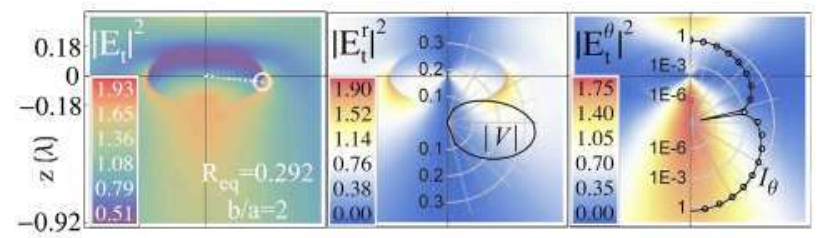

Fig. 4. (Color online) Electric field distributions in the $y z$ plane of a $n_{r}=1.33$ oblate spheroid with size parameter $C=2$ and aspect ratio $b / a=2$. In the $I_{\theta}$ curve the circular dots correspond to the values obtained by Asano and Yamamoto [2], shown for comparison. 


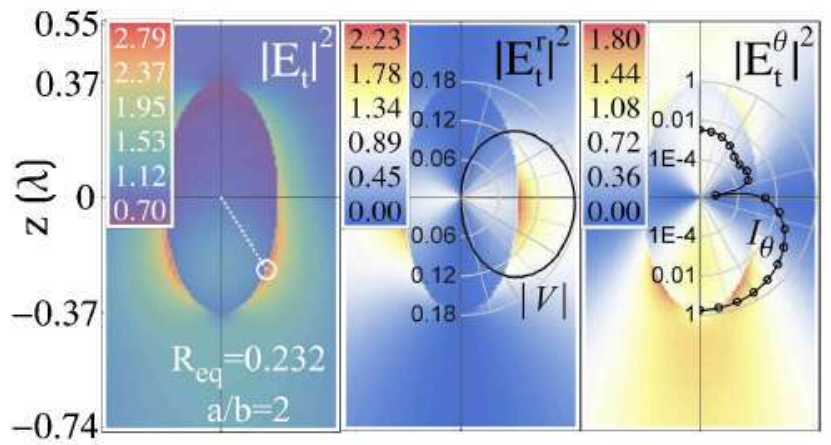

Fig. 5. (Color online) Same as Fig. 4 but for a prolate spheroid with aspect ratio $a / b=2$.

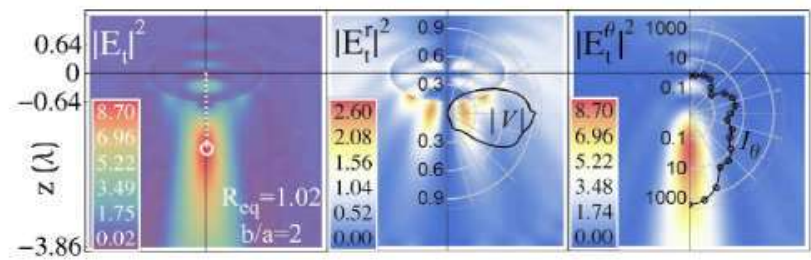

Fig. 6. (Color online) Electric field distributions in the $y z$ plane of a $n_{r}=1.33$ oblate with size parameter $C=7$ and aspect ratio $b / a=2$. In the $I_{\theta}$ curves the circular dots correspond to the values obtained in [2].

\section{B. The Electrostatic Case}

Particles much smaller than $\lambda$ act as electric dipoles generating internal and scattered fields parallel to the incident field. This is known as the ES or non-retarded regime and occurs when the particle size is sufficiently small to satisfy both Rayleigh limit conditions [14],

$$
2 \pi R_{e q} / \lambda \ll 1, \quad\left|n_{r}\right| 2 \pi R_{e q} / \lambda \ll 1,
$$

where $R_{e q}$ is a characteristic length of the particle. For spheres it is simply the radius, and for spheroids $R_{e q}$ is taken as the radius of the volume-equivalent sphere [31]. The refractive index of the particle relative to the medium is taken to be $n_{r}=1.33$.

Even though the present method can accurately calculate scattering by absorbing spheroids (with a nonzero imaginary part in $n_{r}$ ), a purely real refractive index is considered since it facilitates comparison with the previous literature results. Furthermore, it has been verified that the inclusion of a small imaginary part (on the order of $0.001 i$ ) in $n_{r}$. does not significantly change the results and conclusions given here.

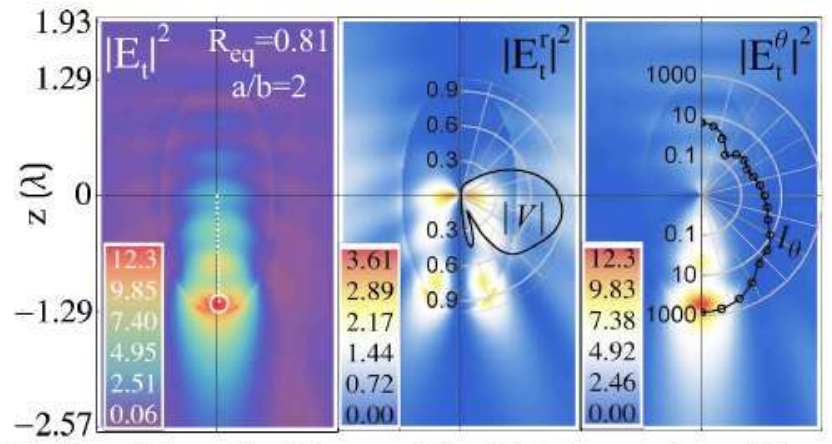

Fig. 7. (Color online) Same as Fig. 6 but for a prolate with aspect ratio $a / b=2$.
As long as conditions (13) are fulfilled the scattered field distribution is the same regardless of the particle size. Figure 3 shows the field distribution inside and in a close vicinity outside a sphere with a radius much smaller than $\lambda$. The plot on the left is the magnitude squared of the total field $\left(\mathbf{E}_{t}\right)$, given by $\mathbf{E}_{\mathbf{t}}=\mathbf{E}_{\mathbf{I}}$ in the interior of the particle and $\mathbf{E}_{\mathbf{t}}=\mathbf{E}_{\mathbf{S}}+\mathbf{E}_{0}$ in the exterior. The central and right plots in Fig. 3 depict the magnitude squared of the longitudinal (along $\mathbf{r}$ ) and transverse (orthogonal to $\mathbf{r}$ ) components of $\mathbf{E}_{\mathbf{t}}$, respectively. If the particle shape were oblate or prolate the only practical change that would occur in Fig. 3 would be in the scale values. The characteristics which we are mainly interested, such as the peak electric field position and polarization, are shapeindependent in the ES regime.

As a dipole, the field inside the particle is uniform and along $\mathbf{E}_{0}$. The external field is highest close to the surface in that direction, where the longitudinal component is dominant. The longitudinal field is evanescent; it decays very rapidly, roughly with $1 / r^{3}$, with $r$ being the distance from the particle center. Since the magnitude of the transverse component only decays with $1 / r$ this becomes the dominant component in the far-field.

In the field distributions shown in this paper the longitudinal and transverse components are represented separately since they have a distinct physical nature. A transverse EM field is a propagating wave; whereas a longitudinal field is, at some time $t$, an instantaneous localized Coulomb field associated with a static charge distribution [32]. This is important to take into account when modeling the interaction between the scattered light and a material placed in the near-field. For instance, when considering the transference of energy from the scattered beam to a medium, one can use the classical Poynting vector $\mathbf{S}=\mathbf{E} \times \mathbf{H}$ for the energy flux of the transverse part of the field (the only component present far away from a scatterer). However, the concept of Poynting vector is not applicable to a non-propagating longitudinal field since it is not an EM wave, as defined classically [33].

Only semi-classical formalisms can account for a reasonable interpretation of light-matter interaction by the longitudinal component [32]. In this way it has been derived [18] that the rate of energy absorption from a longitudinal field, such as that in the near-field of scatterers, should be proportional to the squared magnitude of the scalar potential $V(r)=-\int_{0}^{r} \mathbf{E} \cdot \mathbf{d r}$. Therefore, the plots of the longitudinal $E_{t}^{r}$ component distributions shown in this work include a polar plot of the magnitude of $V(r)$ at the surface of the particle. In Fig. 3 it can be seen that, in the ES regime, the spot of the maximum field intensity coincides with that of the maximum potential at the particle surface. The same is not verified for particles with bigger sizes.

The far-field pattern is usually analyzed by calculating the intensities of the scattered light at infinite distances from the scattering object. In this limit one can take $C_{0} \xi$ $\rightarrow \infty$ and neglect terms with $\xi^{-2}$ and higher order inverse powers of $\xi$ in expression (3). By doing so, the longitudinal/radial terms ( $E^{\xi}$ and $H^{\xi}$ ) vanish and the scattered wave becomes a purely transverse wave. Besides, the angular coordinate $\eta \rightarrow \cos (\theta)$ becomes a function of $\theta$ 
only. As such, the squared magnitude of the scattered (electric) far-field vector in the plane of $\mathbf{E}_{0}(\phi=\pi / 2)$ reduces to [3] $\left|\mathbf{E}_{S}(\phi=\pi / 2)\right|^{2}=I_{\theta}(\theta) /\left(K_{0} r\right)^{2}$, where $I_{\theta}$ is the intensity function on the scattering plane given by

$$
I_{\theta}(\theta)=\left|\sum_{n=0}^{\infty}-i^{n} \pi \alpha_{n} S_{0, n}\left(C_{0}, \cos \theta\right)\right|^{2} .
$$

A polar plot of this quantity is included in the $\left|E_{t}^{\theta}\right|^{2}$ plots presented in this paper, in order to compare the transverse component in the near-and far-fields. For a small scatterer (see Fig. 3 ) the $I_{\theta}$ pattern is approximately proportional to $\cos ^{2}(\theta)$ with equal back and frontal lobestypical of scattering in the Rayleigh limit $[2,14]$. This is similar to the distribution of $\left|E_{t}^{\theta}\right|^{2}$ in the near-field.

Another typical characteristic of the ES regime that can be observed in Fig. 3 is that all quantities are perfectly symmetric with respect to the $y$ axis. This means that in this regime there is no $K$ dependence in the scattering; it only depends on the incident electric field vector $\mathbf{E}_{0}$ [14]. The opposite occurs for particles with large sizes relative to $\lambda$, as shall be shown in the following subsections, where scattering becomes mainly $\mathbf{K}_{0}$ dependent.

\section{The Transition Regime}

When the scatterer size is not much smaller than $\lambda$ the field is no longer uniform in its interior due to the appearance of retarded potentials. In the model, this behavior arises due to the excitation of higher order modes in the fields' expansions (2)-(4). The bigger the particle the more modes come into play [Eq. (6)], causing the appearance of quadrupolar, octopolar, and other features in the field patterns that are highly dependent on the particle geometry.

As explained in the previous subsection, in the small particle (ES) limit the highest scattered field magnitude occurs in the $\mathbf{E}_{0}$ direction at the particle surface. For spheroids with $R_{e q}>\lambda$ the peak field magnitude is on the axis of propagation $\left(\mathbf{K}_{0}\right)$, which is known as forward scattering (FS) [15]. Between the ES and FS cases there is a transition regime for particles with $R_{\ell \varphi}$ sizes above those set by conditions (13), but still smaller than $\lambda$.

This transition regime has unique characteristics since the peak scattered field can lie in between the $\mathbf{E}_{0}$ and $\mathbf{K}_{0}$ axes, depending on the particle size and shape. This can be well illustrated by spheroidal particles with a size parameter $C=2$ [as defined in Eq. (1)]. Figures 4 and 5 show the field distributions produced by oblate and prolate spheroids, respectively, with the size parameter and aspect ratio equal to 2 . Similar calculations were performed for spheroids with higher aspect ratios and with the same size parameter. Due to their lower volume $\left(R_{e q}\right)$ it was observed that the more elongated the spheroid is the more its field distribution resembles that of a dipole in the ES regime.

The near- and far-field quantities in the plots of Figs. 4 and 5 are no longer symmetric with respect to the $y$ axis, hence exhibiting a certain $K$ dependence that was not present in the ES case. The longitudinal field and potential do not peak in the horizontal $\mathbf{E}_{0}$ direction but move down slightly closer to the vertical $\mathbf{K}_{0}$ axis. In the case of the oblate in Fig. 4 the maximum of the transverse component $E_{t}^{\theta}$ is almost as intense as that of the longitudinal
$E_{t}^{r}$ and occurs in the forward direction. However, for the prolate in Fig. 5 the $E_{t}^{\theta}$ near-field distribution has a dominant quadrupolar feature with two frontal lobes peaking at angles of $\pm 32.2^{\circ}$ with the negative $z$ axis. Such a feature is not present in the far-field $I_{\partial}$ pattern.

\section{The Forward Scattering Regime}

In the forward scattering (FS) regime, spheroids whose indices of refraction are real and higher than the medium focus the light in the forward direction, producing scattered fields along their axes of revolution that can be more than 1 order of magnitude higher than the incident field.

Figures 6 and 7 show the field distribution for spheroids with a size parameter $C=7$, in the FS regime. Inside the particles retarded potentials cause the appearance of standing waves. The transversal component $E_{t}^{\theta}$ of these electric waves propagates into the far-field causing the distinct lobes in the $I_{\theta}$ plot. In this size range this component plays the main role even in the near-field since the spot of maximum $\left|E_{t}\right|$ is transverse polarized. This does not occur in the previous regimes for smaller particles where the longitudinal component has the highest intensities close to the scatterer.

The spots of the strongest $E_{f}$ field are quite distinct for the oblate and prolate shapes in Figs. 6 and 7, respectively. The field enhancement achieved with the prolate is higher than with the oblate of the same size, but it is confined to a smaller region at the prolate front edge. The "bright region" in the oblate case is positioned away from the particle surface and extends through a distance in the negative $z$ direction much higher than the particle $R_{e q}$ (about ten times higher as shown below in Subsection 4.B).

The curves of the potential $|V|$ at the surface present additional features to those for smaller particles, owing to the peaks of the radial electric field component $E_{t}^{r}$. Some of these peaks now appear close to the incidence direction, but right at the $z$ axis there is always a minimum in the longitudinal field no matter how big is the particle.

Both spheroids in Figs. 6 and 7 have an aspect ratio of 2. Spheroids with the same size parameter $C=7$ but with an aspect ratio of 5, for instance, have smaller $R_{e q}$ 's. As a result, the field distributions of such elongated particles present the characteristics of the transition regime described in Subsection 3.C). Therefore, it is better to compare field distributions of particles with different aspect ratios that have the same $R_{e q}$.

Figure 8 shows the results of a prolate with the same $R_{\ell q}$ as that of Fig. 7 but more elongated ( $a / b=5$ ). In this case the light enhancement occurs mainly in the interior of the particle and in a localized region close to the surface. The spatial extent of the maximum field peak (signaled with the white circle) is even smaller than in Fig. 7. The plot of the total field $\left|E_{t}\right|^{2}$ is almost identical to that of $\left|E_{\ell}\right|^{2}$, with the longitudinal component only being relevant in a small dipole-like feature close to the particle center.

\section{DISCUSSION OF RESULTS}

A. Direction of Maximum Scattering

The field distributions shown in Figs. 4-8 correspond to a few selected geometries that illustrate the particularities 


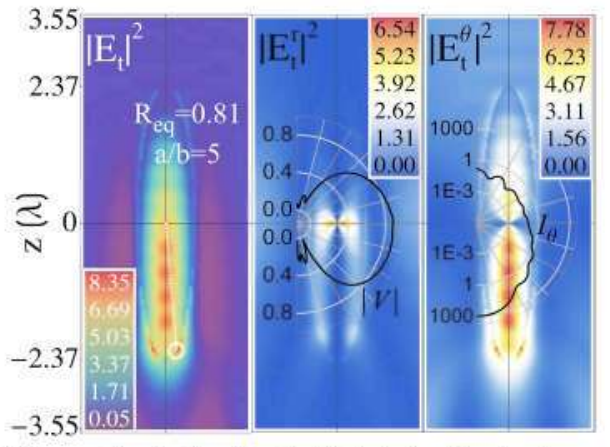

Fig. 8. (Color online) Electric field distribution in the $y z$ plane of a $n_{r}=1.33$ spheroid with size parameter $C=14.6$. This is an elongated prolate with aspect ratio $a / b=5$ and the same $R_{e q}$ as the prolate in Fig. 7.

of light scattering by spheroids with sizes on the order of the incident wavelength $\lambda$. In this section these characteristics are analyzed in more detail. We start by looking at the $\theta$-angle at which the highest field intensity occurs $\left(\theta_{\mathrm{MAX}}\right)$. This quantity is a good indicator of the distinct scattering regimes described in Section 3 as can be seen in the plot of Fig. 9. The plot represents the value of $\theta_{\mathrm{MAX}}$ as a function of the spheroid size $R_{e q}$.

In the ES case, the direction of highest field strength is always the same as the incident $E$-field direction $\left(\theta_{\text {MAX }}\right.$ $=90^{\circ}$ ), independently of the scatterer. Spheroids with $R_{e q} \cong \lambda$, or higher, also have a fixed peak scattering direction which is the incidence direction $\left(\theta_{\operatorname{MAX}}=0^{\circ}\right)$, regardless of their shape-the FS regime. Between these extremes there is a transition regime where the direction of highest field enhancement switches according to the spheroid size and shape. This interval of particle sizes where $\theta_{\text {MAX }}$ can lie between $90^{\circ}$ and $0^{\circ}$ is broader for prolate spheroids and narrower for oblates. The more elongated is the oblate the more abrupt is the transition from $\theta_{\mathrm{MAX}}=90^{\circ}$ to $\theta_{\mathrm{MAX}}=0^{\circ}$.

Figure 9 also shows the case of spheres $(a / b=1)$ calculated with Mie theory. The transition regime for spheres

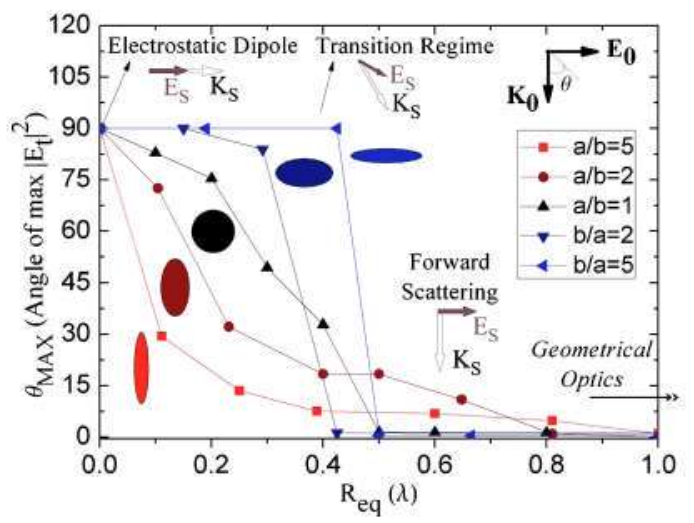

Fig. 9. (Color online) Angle $\theta$ (in degrees) between the incidence direction and the position of maximum field enhancement. In the plots of Figs. 3-8 this is the angle between the white dashed line and the negative $z$ axis. The schematic $E_{S}$ and $K_{S}$ vectors represent the polarization of the scattered field at the spot of maximum field intensity. From the dipole to the FS regime the polarization goes from being purely longitudinal to transverse, passing by a transition regime where it has both longitudinal and transverse components. occurs between $R_{e q} \ll \lambda$ and $R_{e q} \approx \lambda / 2$; and in that interval $\theta_{\text {MAX }}$ decreases roughly linearly with $R_{e q}$.

The peak scattered field in the dipole case is dominantly longitudinal and occurs at the particle surface. This longitudinal component evanesces rapidly with the distance from the particle, so in the far-field only the transverse part of the scattered wave is present.

As the particle size increases and we approach the FS regime, the transverse component becomes progressively more dominant relative to the longitudinal even in close proximity to the particle. So for big sizes the scattered field is mainly transverse both in the near and far regions as illustrated in Figs. 6-8. In the transition regime the longitudinal and transverse components have similar magnitudes in the near-field as observed in Figs. 4 and 5.

\section{B. Extension of Field Enhancement}

Spheroidal particles with a real index of refraction higher than that of the surrounding medium act as lenses in the FS regime, concentrating the light in a bright region which can be located inside or outside the particle but always in the incidence axis. According to the principles of physical optics [30], a particle in this regime acts as a resonator (cavity) for the light that is transmitted to its interior. Inside the particle some of that light is internally reflected, bouncing back and forth at distinct angles with the surface. This produces the appearance of an interference pattern both in the internal and external fields as can be seen in the images of Subsection 3.D. The electric field peaks occur at the spots where the light rays interfere more constructively.

Figure 10 shows the spatial extension of the bright region of field amplification, where the maximum $E_{t}$ is located, for spheroids with the aspect ratios analyzed in this work and $R_{e q}$ sizes close to $\lambda$. The curves in the figure correspond to the values of $\left|\mathbf{E}_{t}\right|^{2}$ along the radial line

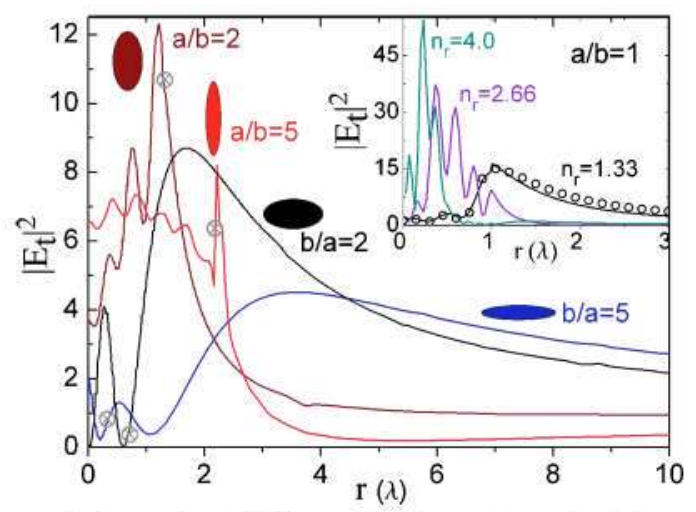

Fig. 10. (Color online) $\left|\mathbf{E}_{t}\right|^{2}$ profiles along the radial direction of maximum field $\left(\theta_{\mathrm{MAX}}\right)$, represented by the white dashed line in the plots of Section 3, for particles with size close to $\lambda . r$ is the distance to the particle center, in units of $\lambda$. The spheroids with aspect ratio 2 correspond to those in Figs. 6 and 7. The prolate with $a / b=5$ corresponds to Fig. 8 . The oblate with $b / a=5$ has the same $R_{e q}$ as the oblate with $b / a=2$. The gray crossed circles represent the intersection with the particle surface. The inset shows similar $\left|\mathbf{E}_{t}\right|^{2}$ profiles for spheres with radius $R=\lambda$ but distinct refractive indices $n_{r}$, computed with Mie theory. The curve for $n_{r}$ $=1.33$ is compared with that (circular dots) calculated with the method described in this work using an almost spherical prolate $(a / b=1.001)$. 
starting at the particle center and passing by the point of maximum enhancement (represented in the images of Section 3 by a white circle).

It can be observed that prolates produce more localized field amplifications, positioned inside the particle and on the outside close to the surface. In the exterior of the prolate this field enhancement decays very rapidly with the distance, and the higher is the elongation of the prolate the more rapidly it decays. On the other hand, the peak scattered intensity produced by oblates is located outside the particle away from the surface and decays very slowly with the distance. For instance, the region of field amplification close to the oblate with $b / a=2$ extends to a distance above ten times the particle $R_{e q}$. The higher is the oblate aspect ratio the lower is the slope of the $E_{t}$ decay.

The spheroids considered in this work have a real index of refraction of 1.33 relative to the medium. Increasing the particle refractive index causes more bending of the light rays transmitted to the particle material, increasing the interference in its interior. Therefore, the higher is the particle real refractive index, the higher the peak field magnitude is expected to be. This is shown in the inset of Fig. 10 for spheres with radius equal to $\lambda$ and distinct relative refractive indices. It can be seen that bigger indices cause an increase in the maximum $E_{f}$ and shift the field enhancement closer to the particle center.

\section{CONCLUSIONS AND FINAL REMARKS}

A separation-of-variables method is used to solve the scattering problem of a spheroidal particle illuminated by an axially incident plane-wave. This method can be extended to the case of an arbitrary excitation beam using the generalized Lorenz-Mie theory [34].

Depending on the characteristic size of the scattering particle $\left(R_{e q}\right)$ relative to the illumination wavelength three different regimes can be distinguished: electrostatic (ES) $\left(R_{e q} \ll \lambda\right)$, intermediate $\left(R_{e q} \sim \lambda\right)$, and geometrical optics $\left(R_{e q} \gg \lambda\right)$. In this paper we focus on the intermediate regime for particles with a real index of refraction of 1.33 times that of the surrounding medium. It is seen that this regime can be subdivided into two which are called the transition and forward scattering (FS) regimes.

The internal field and near-field around spheroids in these size regimes exhibit peculiar characteristics that can be of interest for distinct technological applications. According to the particle size and shape, the internal field and external near-field can achieve intensities 1 or more orders of magnitude higher than the incident intensity. Furthermore, the spatial location of the field enhancement can be controlled with the spheroidal geometry. This possibility does not exist in any of the other size regimes: small particles in the ES case produce a peak scattered field in the direction of the incident field and at the particle surface, and big particles scatter the light mainly in the direction of incidence. However, with wavelengthsized spheroids the maximum intensity direction can be located anywhere between the directions of the previous two cases, and it can occur closer to the particle center for prolates and away from it for oblates.

The spatial extension over which the field enhancement is felt is another parameter that can be switched ac- cording to the spheroid geometry. In the FS regime, prolate shapes produce confined enhancements in their interior or in the vicinity of the surface, whereas oblates exhibit peak scattered fields in their exterior which can extend to a distance several times higher than its size (see Fig. 10).

Metallic scatterers sustaining localized surface plasmons can also produce very high light amplification in the ES regime $[11,18]$. In the intermediate regime the performance of metallic particles for the field enhancement is deteriorated because their absorption becomes higher than their scattering power, so they mainly shade the incoming light. Therefore, in this size regime, dielectric scatterers with a real index of refraction higher than the medium perform better. In the cases analyzed here, high field enhancements are observed with dielectric scatterers having only a $33 \%$ difference between the particle and medium refractive indices. This is a crucial advantage of working with dielectric particles in this size regime, since for certain applications it may not be beneficial to have a high material contrast. For instance, for solar cell applications [11] the introduction of metals inside the active material can degrade the device performance since metals cause severe electron-hole recombination. Also, for biological applications, like cancer ablation or in vivo imaging, the introduction of dielectric particles may cause less harm to the organism than metallic ones.

\section{APPENDIX}

The spheroidal coordinates $(\eta, \xi, \phi)$ are related to the Cartesian coordinates by the following transformations:

$$
\begin{gathered}
x=\frac{d}{2} \sqrt{\left(1-\eta^{2}\right)\left(\xi^{2} \pm 1\right)} \cos \phi, \\
y=\frac{d}{2} \sqrt{\left(1-\eta^{2}\right)\left(\xi^{2} \pm 1\right)} \sin \phi, \\
z=\frac{d}{2} \eta \xi,
\end{gathered}
$$

where the upper and lower signs pertain to oblate and prolate spheroidal coordinates, respectively, and $d$ is the interfocal distance. The domains of the spheroidal coordinates are

$$
-1 \leq \eta \leq 1, \quad \varepsilon \leq \xi<\infty, \quad 0 \leq \phi<2 \pi,
$$

where $\varepsilon=0$ in the oblate case and $\varepsilon=1$ in the prolate.

The expressions of the spheroidal vector wave functions used in Eqs. (2)-(4) are given below [3,19]:

$$
\begin{aligned}
\mathbf{M}_{e 0 n}^{x(j)}(C ; \eta, \xi, \phi): & \\
M_{e(i n, \eta}^{x i j)} & =-\frac{2\left(\xi^{2} \pm 1\right)^{1 / 2}}{d\left(\xi^{2} \pm \eta^{2}\right)^{1 / 2}} S_{0 n} \frac{\mathrm{d}}{\mathrm{d} \xi} R_{(m)}^{(i)} \sin \phi, \\
M_{e 0 n, \xi}^{x(i)} & =\frac{2\left(1-\eta^{2}\right)^{1 / 2}}{d\left(\xi^{2} \pm \eta^{2}\right)^{1 / 2}} \frac{\mathrm{d}}{\mathrm{d} \eta} S_{0 n} R_{0 n}^{(j)} \sin \phi,
\end{aligned}
$$




$$
\begin{aligned}
M_{e j), \phi}^{x i j)}= & \frac{2}{d\left(\xi^{2} \pm \eta^{2}\right)}\left[\xi\left(1-\eta^{2}\right) \frac{\mathrm{d}}{\mathrm{d} \eta} S_{0)} R_{(h)}^{(i j)}\right. \\
& \left.+\eta\left(\xi^{2} \pm 1\right) S_{0) n} \frac{\mathrm{d}}{\mathrm{d} \xi} R_{0 n}^{(i)}\right] \cos \phi .
\end{aligned}
$$

$\mathbf{N}_{e, n}^{x i i}(C ; \eta, \xi, \phi):$

$$
\begin{aligned}
& N_{e 0 n, \eta \eta}^{x f i r}=\frac{4}{K d^{2}\left(\xi^{2} \pm \eta^{2}\right)^{1 / 2}}\left[\eta S_{0 n} \frac{\partial}{\partial \xi}\left(\frac{\left(\xi^{2} \pm 1\right)^{3 / 2}}{\left(\xi^{2} \pm \eta^{2}\right)} \frac{\mathrm{d}}{\mathrm{d} \xi} R_{0 n}^{(i)}\right)\right. \\
& -\frac{1}{\left(\xi^{2} \pm 1\right)^{1 / 2}} \frac{\mathrm{d}}{\mathrm{d} \eta} S_{0)} R_{0) n}^{(i)}+(1 \\
& \left.\left.-\eta^{2}\right) \frac{\mathrm{d}}{\mathrm{d} \eta} S_{(n z} \frac{\partial}{\partial \xi}\left(\frac{\xi\left(\xi^{2} \pm 1\right)^{1 / 2} R_{0 n}^{(i)}}{\xi^{2} \pm \eta^{2}}\right)\right] \cos \phi, \\
& N_{e 0 n, \xi}^{x(i)}=-\frac{4}{K d^{2}\left(\xi^{2} \pm \eta^{2}\right)^{1 / 2}}\left[\xi \frac{\partial}{\partial \eta}\left(\frac{\left(1-\eta^{2}\right)^{3 / 2}}{\left(\xi^{2} \pm \eta^{2}\right)} \frac{\mathrm{d}}{\mathrm{d} \eta} S_{0 n}\right) R_{0 n}^{(i)}\right. \\
& +\frac{1}{\left(1-\eta^{2}\right)^{1 / 2}} S_{(n t} \frac{\mathrm{d}}{\mathrm{d} \xi} R_{(h)}^{(i)} \\
& \left.+\left(\xi^{2} \pm 1\right) \frac{\partial}{\partial \eta}\left(\frac{\eta\left(1-\eta^{2}\right)^{1 / 2} S_{0 n}}{\xi^{2} \pm \eta^{2}}\right) \frac{\mathrm{d}}{\mathrm{d} \xi} R_{0 n}^{(i)}\right] \cos \phi, \\
& N_{e 0 n, \phi}^{x(i)}=\frac{4\left(1-\eta^{2}\right)^{1 / 2}\left(\xi^{2} \pm 1\right)^{1 / 2}}{K d^{2}\left(\xi^{2} \pm \eta^{2}\right)}\left[\frac{1}{\left(\xi^{2} \pm 1\right)^{1 / 2}} \frac{\partial}{\partial \eta}((1\right. \\
& \left.\left.-\eta^{2}\right)^{1 / 2} \frac{\mathrm{d}}{\mathrm{d} \eta} S_{0 n}\right) R_{0 n}^{(i)} \\
& \left.+\frac{1}{\left(1-\eta^{2}\right)^{1 / 2}} S_{(h t} \frac{\partial}{\partial \xi}\left(\left(\xi^{2} \pm 1\right)^{1 / 2} \frac{\mathrm{d}}{\mathrm{d} \xi} R_{(h)}^{(i)}\right)\right] \sin \phi .
\end{aligned}
$$

$\mathbf{M}_{e 1 n}^{z(j)}(C ; \eta, \xi, \phi):$

$$
\begin{aligned}
M_{e i n, \eta}^{(i j)}= & \frac{2 \eta}{d\left(\xi^{2} \pm \eta^{2}\right)^{1 / 2}\left(1-\eta^{2}\right)^{1 / 2}} S_{1 n} R_{1 n}^{(i)} \sin \phi, \\
M_{e 1 n, \xi}^{z(i)}= & -\frac{2 \xi}{d\left(\xi^{2} \pm \eta^{2}\right)^{1 / 2}\left(\xi^{2} \pm 1\right)^{1 / 2}} S_{1 n} R_{1 n}^{(i)} \sin \phi, \\
M_{e 1 n, \phi}^{z(i)}= & \frac{2\left(\xi^{2} \pm 1\right)^{1 / 2}\left(1-\eta^{2}\right)^{1 / 2}}{d\left(\xi^{2} \pm \eta^{2}\right)}\left[\eta \frac{\mathrm{d}}{\eta} \frac{\mathrm{d} \eta}{\mathrm{d}} S_{1 n} R_{1 n}^{(i)}\right. \\
& \left.-\xi S_{1 n} \frac{\mathrm{d}}{\mathrm{d} \xi} R_{1 n}^{(i)}\right] \cos \phi .
\end{aligned}
$$

$$
\begin{aligned}
& \mathbf{N}_{e .1 n}^{z(i)}(C ; \eta, \xi, \phi): \\
& N_{e 1 \eta, \eta}^{(i j)}=\frac{4\left(1-\eta^{2}\right)}{K d^{2}\left(\xi^{2} \pm \eta^{2}\right)^{1 / 2}}\left[\eta \frac{\mathrm{d}}{\mathrm{d} \eta} S_{1 n} \frac{\partial}{\partial \xi}\left(\frac{\xi^{2} \pm 1}{\xi^{2} \pm \eta^{2}} R_{1 \eta}^{(i j)}\right)\right. \\
& -S_{1 n} \frac{\partial}{\partial \xi}\left(\frac{\xi\left(\xi^{2} \pm 1\right)}{\xi^{2} \pm \eta^{2}} \frac{\mathrm{d}}{\mathrm{d} \xi} R_{1 n}^{(i)}\right) \\
& \left.+\frac{\xi}{\left(1-\eta^{2}\right)\left(\xi^{2} \pm 1\right)} S_{1 n} R_{1 n}^{(i)}\right] \cos \phi, \\
& N_{\varepsilon i n, \xi}^{z(i)}=\frac{4\left(\xi^{2} \pm 1\right)^{1 / 2}}{K d^{2}\left(\xi^{2} \pm \eta^{2}\right)^{1 / 2}}\left[\frac{\partial}{\partial \eta}\left(\frac{1-\eta^{2}}{\xi^{2} \pm \eta^{2}} S_{1 n}\right) \frac{\mathrm{d}}{\mathrm{d} \xi} R_{1 n}^{(i)}\right. \\
& -\frac{\partial}{\partial \eta}\left(\frac{\eta\left(1-\eta^{2}\right)}{\xi^{2} \pm \eta^{2}} \frac{\mathrm{d}}{\mathrm{d} \eta} S_{1 n}\right) R_{1 n}^{(i)} \\
& \left.+\frac{\eta}{\left(1-\eta^{2}\right)\left(\xi^{2} \pm 1\right)} S_{1 n} R_{1 n}^{i j j}\right] \cos \phi, \\
& N_{e 1 n, \varphi}^{z i r}=-\frac{4\left(1-\eta^{2}\right)^{1 / 2}\left(\xi^{2} \pm 1\right)^{1 / 2}}{K d^{2}\left(\xi^{2} \pm \eta^{2}\right)}\left[\frac{\xi}{\xi^{2} \pm 1} \frac{\mathrm{d}}{\mathrm{d} \eta} S_{1 n} R_{1 n}^{(i)}\right. \\
& \left.+\frac{\eta}{1-\eta^{2}} S_{1 n} \frac{\mathrm{d}}{\mathrm{d} \xi} R_{1 n}^{(i)}\right] \sin \phi \text {. }
\end{aligned}
$$

\section{ACKNOWLEDGMENTS}

We are grateful to Professor Le-Wei $\mathrm{Li}$ for providing the numerical routines developed by his group [3]. This work has been supported by the IBPOWER grant No. 211640 of the European Commission, by the GENESIS FV grant No. CSD2006-0004 of the Spanish program CONSOLIDER, and by the NUMANCIA grant No. S-0505/ ENE/0310 of Comunidad de Madrid. M. J. Mendes also acknowledges the Portuguese Government grant No. SFRH/BD/21669/2005 of the Fundação para a Ciência e Tecnologia (Ministério da Ciência, Tecnologia e Ensino Superior) (FCT-MCTES).

\section{REFERENCES}

1. G. Mie, "Articles on the optical characteristics of turbid tubes, especially colloidal metal solutions," Ann. Phys. (Berlin) 25, 37 7-445 (1908).

2. S. Asano and G. Yamamoto, "Light-scattering by a spheroidal particle," Appl. Opt. 14, 29-49 (1975).

3. L.-W. Li, X.-K. Kang, and M.-S. Leong, Spheroidal Wave Functions in Electromagnetic Theory (Wiley, 2002).

4. J. A. Morrison and M. J. Cross, "Scattering of a plane electromagnetic-wave by axisymmetric raindrops," Bell Syst. Tech. J. 53, 955-1019 (1974).

5. B. P. Sinha and R. H. Macphie, "Electromagnetic scattering by prolate spheroids for plane-waves with arbitrary polarization and angle of incidence," Radio Sci. 12, 171-184 (1977).

6. A. R. Sebak and B. P. Sinha, "Scattering by a conducting spheroidal object. with dielectric coating at axial incidence," IEEE Trans. Antennas Propag. 40, 268-274 (1992).

7. C. Girard and A. Dereux, "Near-field optics theories," Rep. Prog. Phys. 59, 657-699 (1996).

8. L. Novotny and B. Hecht, Principles of Nano-Optics (Cambridge Univ. Press, 2006).

9. L. A. Blanco and F. J. García de Abajo, "Spontaneous emis- 
sion enhancement near nanoparticles," J. Quant. Spectrosc. Radiat. Transf. 89, 37-42 (2004).

10. M. P. Cline, P. W. Barber, and R. K. Chang, "Surfaceenhanced electric intensities on transition- and noble-metal spheroids," J. Opt. Soc. Am. B 3, 15-21 (1986).

11. M. J. Mendes, A. Luque, I. Tobias, and A. Marti, "Plasmonic light enhancement in the near-field of metallic nanospheroids for application in intermediate band solar cells," Appl. Phys. Lett. 95, 071105 (2009).

12. C. H. Li, G. W. Kattawar, P. W. Zhai, and P. Yang, "Electric and magnetic energy density distributions inside and outside dielectric particles illuminated by a plane electromagnetic wave," Opt. Express 13, 4554-4559 (2005).

13. E. S. Thiele and R. H. French, "Light-scattering properties of representative, morphological mitile titania particles studied using a finite-element method," in 99th Annual Meeting of the American-Ceramic-Society (American Ceramic Society, 1997), pp. 469-479.

14. C. F. Bohren and D. R. Huffman, Absorption and Scattering of Light by Small Particles (Wiley-VCH, 2004).

15. M. Born and E. Wolf, Principles of Optics: Electromagnetic Therly of Propagation, Interference and Diffraction of Light, 7th ed. (Cambridge Univ. Press, 1999).

16. J. P. Barton, "Internal, near-surface, and scattered electromagnetic fields for a layered spheroid with arbitrary illumination," Appl. Opt. 40, 3598-3607 (2001).

17. T. Oguchi, "Attenuation and phase rotation of radio-waves due to rain-calculations at 19.3 and $34.8 \mathrm{GHz}$," Radio Sci 8, 31-38 (1973)

18. A. Luque, A. Marti, M. J. Mendes, and I. Tobias, "Light absorption in the near field around surface plasmon polaritons," J. Appl. Phys. 104, 113118 (2008)

19. C. Flammer, Spheroidal Wave Funxtions (Stanford U. Press, 1957).

20. P. E. Falloon, P. C. Abbott, and J. B. Wang, "Theory and computation of spheroidal wavefunctions," J. Phys. A 36, 5477-5495 (2003).

21. L. W. Li, M. S. Leong, T. S. Yeo, P. S. Kooi, and K. Y. Tan, "Computations of spheroidal harmonics with complex arguments: a review with an algorithm," Phys. Rev. E 58, 6792 6806 (1998)

22. M. Abramowitz and I. A. Stegun, Handbook of Mathemati. cal Functions (Dover, 1970).
23. D. B. Hodge, "Eigenvalıes and eigenfinctions of spheroidal wave equation," J. Math. Phys. 11, 2308-2312 (1970).

24. W. Press, B. Flannery, S. Teukolsky, and W. Vetterling, $N u$. merical Recipes in FORTRAN 77: The Art of Scientific Com. puting (Cambridge Univ. Press, 1992).

25. K. S. Joo and M. F. Iskander, "A new procedure of pointmatching method for calculating the absorption and scattering of lossy dielectric objects," IEEE Trans. Antennas Propag. 38, 1483-1490 (1990).

26. V. G. Farafonov and V. B. Il'in, "Scattering of light by axially symmetric particles: modification of the point-matching method," Opt. Spectrosc. 100, 437-447 (2006).

27. L. Boyde, K. J. Chalut, and J. Guck, "Interaction of Gaussian beam with near-spherical particle: an analyticnumerical approach for assessing scattering and stresses," J. Opt. Soc. Am. A 26, 1814-1826 (2009).

28. M. Nishimura, S. Takamatsu, and H. Shigesawa, "A numerical analysis of electromagnetic scattering of perfect conducting cylinders by means of discrete singularity method improved by optimization process," Electron. Commun. Jpn. 67, 75-81 (1984).

29. A. C. Ludwig, "A comparison of spherical wave boundaryvalue matching versus integral-equation scattering solutions for a perfectly conducting body," IEEE Trans. Antennas Propag. 34, 857-865 (1986)

30. J. C. Ravey and P. Mazeron, "Light-scattering in the physical optics approximation-application to large spheroids," J. Opt. (Paris) 13, 273-282 (1982).

31. V. V. Somsikov and N. V. Voshchinnikov, "On the applicability of the Rayleigh approximation for coated spheroids in the near-infrared," Astron. Astrophys. 345, 315-320 (1999).

32. C. Cohen-Tannoudji, J. Dupont-Roc, and G. Grynberg, Photons and Atoms-Introduction to Quantum Electrodynamics (Wiley-Interscience, 1997).

33. O. Keller, "Near-field photon wave mechanics in the Lorenz gauge," Phys. Rev. A 76, 062110 (2007).

34. F. Xi1, K. F. Ren, G. Gonesbet, G. Grehan, and X. S. Cai, "Generalized Lorenz-Mie theory for an arbitrarily oriented, located, and shaped beam scattered by a homogeneolis spheroid," J. Opt. Sox. Am. A 24, 119-131 (2007). 